\title{
Misdiagnosis of breast implant rupture with mammography
}

\author{
Bert Van Brenk BSc MD, James L Mahoney MD FRCSC \\ Division of Plastic Surgery, St Michael's Hospital, Toronto, Ontario
}

\begin{abstract}
B Van Brenk, JL Mahoney. Misdiagnosis of breast implant rupture with mammography. Can J Plast Surg 1998;6(1):17-18.

Diagnosis of intracapsular ruptures of silicone breast implants with mammography can be difficult. A case is presented where mammography was used to diagnose an implant rupture with both a false positive and false negative result in the same patient. The mammographic features of breast implant ruptures are discussed.
\end{abstract}

Key Words: Breast implants, Mammography, Rupture, Silicone

\section{Diagnostic erroné de rupture de prothèse mammaire à la mammographie}

RÉSUMÉ : Le diagnostic de rupture intracapsulaire est parfois difficile à poser au moyen de la mammographie dans le cas des prothèses mammaires à la silicone. On présente ici un cas où la mammographie a été utilisée pour un diagnostic de rupture de prothèse ayant donné des résultats faussement positifs et faussement négatifs chez une même patiente. Les caractéristiques mammographiques des ruptures de prothèses mammaires sont décrites dans le présent article.

$\mathrm{M}$ any women who have previously undergone breast augmentation with silicone breast implants are presenting with concerns about rupture of their implants and leakage of silicone. Clinical diagnosis of implant ruptures based on physical findings can be difficult (1). As a result, much emphasis has been placed on diagnosing ruptures of breast implants radiologically. Samuels et al (2) reviewed the literature concerning the sensitivity and specificity of various radiographical techniques for detecting breast implant ruptures. They reported a sensitivity of $45 \%$ to $67 \%$ and a specificity of $50 \%$ to $85 \%$ for detecting ruptures with mammography. Other studies by Bassett et al (3) and Robinson et al (4) suggested that the sensitivity for detecting implant rupture with mammography is even lower, in the range of $11 \%$ to $16 \%$. This translates into significant false negative and false positive rates when using mammography to diagnose implant rupture. We report a case from our institution where there was both a false negative and false positive in the same patient when an implant rupture was diagnosed with mammography.

Correspondence and reprints: Dr JL Mahoney, Division of Plastic Surgery, St Michael's Hospital, 30 Bond Street, Room 420B, Toronto, Ontario M5B 1W8. Telephone 416-864-5385, fax 416-864-5888, e-mail james.mahoney@utoronto.ca

\section{CASE PRESENTATION}

A 56-year-old woman presented with concerns about the status of her breast implants. She had undergone bilateral breast augmentation with silicone gel, saline double-lumen implants approximately 10 years earlier. She was concerned that the left breast implant was ruptured. A routine mammogram had been done at an outside institution and was reviewed with a radiologist at our institution. The mammogram showed folding of the anterior and lateral aspects of the left breast implant, with separation of the inner and outer layers. There was no evidence of radio-opaque material, consistent with silicone, lying outside the margins of the implant (Figure 1). It was thought that the left breast implant was ruptured because there was marked involution of the implant margin, which is consistent with a loss of implant integrity. There was a small area of herniation of the right breast implant posteriorly, but there was no other evidence of implant rupture, and it was believed that the implant was intact (Figure 2). The patient had undergone several closed capsulotomies by the surgeon who placed the implants, but there was no other history of trauma. The patient had not noticed any change in her breasts. She was otherwise healthy except for occasional stiffness in the shoulders and upper back. On examination, there was no obvious breast deformity. The left breast felt softer than the right breast, and the right breast was tender to 


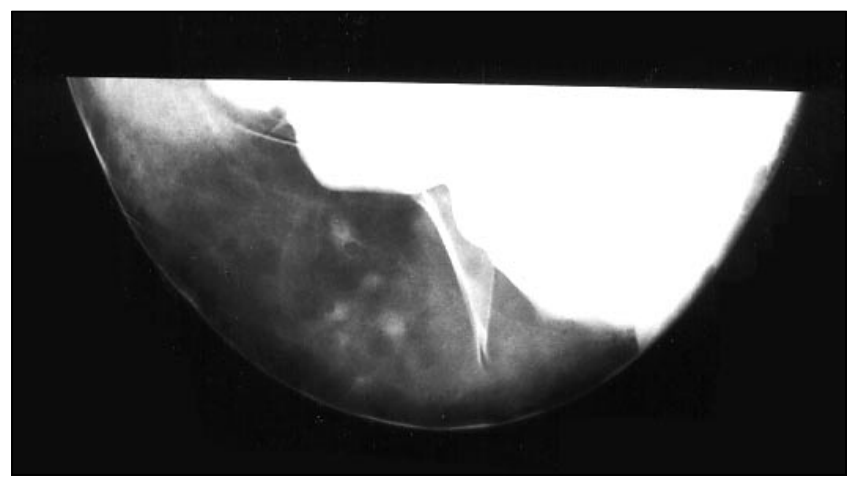

Figure 1) Marked folding of the left breast implant margin

palpation. The patient was advised, and she consented to removal of both breast implants. The patient did not wish to have the implants replaced with saline implants.

At the time of surgery, upon opening the right breast capsule, silicone gel was evident outside the breast implant, obviously due to rupture of the implant. The right breast implant was removed, and a partial capsulectomy was done without difficulty. The left breast implant, having no evidence of rupture, was removed.

\section{DISCUSSION}

Physical findings of a ruptured breast implant are most commonly a change in the size or shape of the breast, a softer feel to the breast, asymmetry and occasionally tenderness (1). These findings may be vague and make diagnosis of a rupture difficult.

Mammography is usually the first screening test used to evaluate the status of implants. Both the literature and this case illustrate that mammography can be inaccurate in evaluating the status of implants. Intracapsular rupture can be quite difficult to diagnose, and mammography is more accurate in detecting extracapsular rupture. Similar densities of the silicone gel and the elastomer envelope may obscure subtle implant changes $(2,5)$. Two mammographic findings that are highly suggestive of extracapsular implant rupture are silicone globules lying outside the margins of the implant and large herniation of the implant (6). Other findings that may indicate implant rupture are irregularity of the implant margins and variability in the density of the implant contents. Ir-

\section{REFERENCES}

1. Andersen B, Hawtof D, Alani H, Kapetansky D. The diagnosis of ruptured breast implants. Plast Reconstr Surg 1989;84:903-7.

2. Samuels JB, Rohrich RJ, Weathrall PT, Ho AM, Goldberg KL. Radiographic diagnosis of breast implant rupture: Current status and comparison of techniques. Plast Reconstr Surg 1995;96:865-77.

3. Bassett LW, Brenner RJ. Consideration when imaging women with breast implants. AMJ Am J Roentgenol 1992;159:979-83.

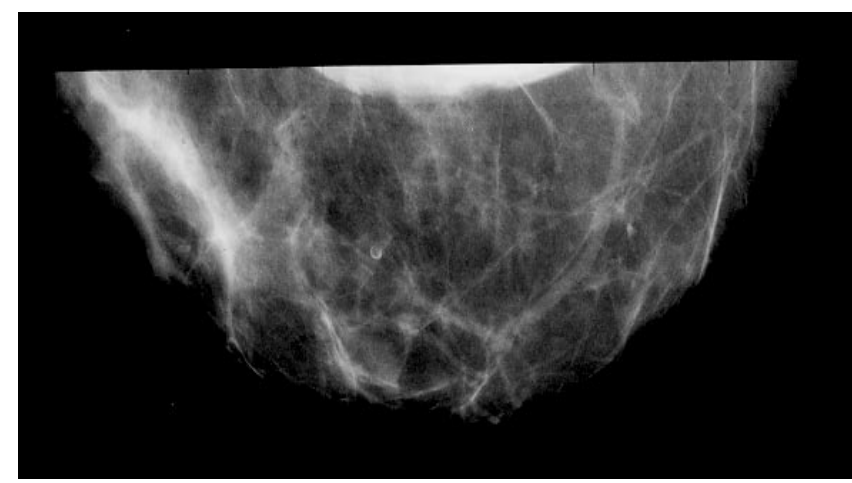

Figure 2) Normal mammogram of the right breast implant

regularity of the implant margin may indicate capsular contraction rather than implant rupture (2). In the case of silicone gel, saline double-lumen implants, irregularity of the capsule may be due to variability in the density of the silicone gel and the saline, and the overlap of these materials during imaging. In this case, there were no mammographic changes suggesting rupture of the right breast implant, even through there was evidence of an intracapsular rupture at surgery.

\section{SUMMARY}

Various radiographical techniques have been used to detect breast implant rupture. Given the reported sensitivity and specificity of mammography in detecting implant rupture, false negatives and positives can result, particularity for intracapsular rupture. The accuracy in detecting extracapsular rupture with mammography is $90 \%$, based on findings that include implant herniation or silicone globules lying outside the implant margins. Intracapsular rupture is much more difficult to detect. Irregularity of the implant margin may indicate intracapsular rupture but may also be due to capsular contraction or variability in the density of the implant materials. In other cases, subtle changes to the implant margin may not be picked up on mammography. If large implant herniation or silicone globules outside the implant margin are not present, an intracapsular implant rupture could still be present. Likewise, irregularity of the implant margin or shape may not represent an implant rupture. In either case, the actual status of the implant can only be identified during surgery.

4. Robinson OG Jr, Bradley EL, Wilson DS. Analysis of explanted silicone implants: A report of 300 cases. Ann Plast Surg 1995;34:1-6.

5. Chung KC, Wilkins EG, Beil RJ Jr, et al. Diagnosis of silicone gel implant rupture by ultrasonography. Plast Reconstr Surg 1996;97:104-9.

6. Peters W, Smith D, Grosman H, Fornasier V. Role of mammography to assess complications of silicone gel breast implants. Can J Plast Surg 1995;3:150-6. 